\title{
Kidney-sparing Management Versus Nephroureterectomy for Upper Tract Urothelial Carcinoma: a Systematic Review and Meta-analysis
}

\author{
You Luo ${ }^{\star}$, Dong-Li She ${ }^{\star}$, Hu Xiong ${ }^{\star}$, Sheng-Jun Fu, Li Yang*
}

\begin{abstract}
Purpose: To evaluate and update evidence for prognostic effects of kidney-sparing (KS) management and nephroureterectomy (NU) for upper tract urothelial carcinomas. Materials and Methods: Pubmed, Embase and the Cochrane Library were retrieved for the identification of comparative studies of kidney-sparing procedure and nephroureterectomy for upper tract urothelial carcinoma prior to December 2014. The data were extracted independently by 2 reviewers and the quality of the included studies was assessed. Review Manager 5.3 and STATA 13 were used to perform the meta-analysis. Results: Twenty-three observational studies including $1,587 \mathrm{KS}$ and $3,996 \mathrm{NU}$ were evaluated. The results of the meta-analysis showed that nephroureterectomy had no significant benefit with regard to intravesical recurrence (IRFS), metastasis (MFS), cancer specific survival (CSS) and overall survival (OS) except the total tumor recurrence (RFS) when compared with kidney sparing management. The respectively pooled outcomes were HR $1.36(0.69-2.68, P=0.38)$ for IRFS, $1.09(0.59-2.01, P=0.78)$ for MFS, 1.17 $(0.77-1.79, P=0.47)$ for CSS, $1.50(0.90-2.48, P=0.12)$ for OS and 1.61 (1.03-2.51, $P=0.04)$ for RFS. Conclusions: On the whole, kidney-sparing management had equivalent prognostic effect on upper tract urothelial carcinoma as the standard nephroureterectomy except in tumor recurrence. However, the results should be interpreted with caution for lack of stage and grade stratification and multi-center randomized controlled trials are still needed to verify our results.
\end{abstract}

Keywords: Upper tract urothelial carcinoma - kidney-sparing management - nephroureterectomy - meta-analysis

Asian Pac J Cancer Prev, 16 (14), 5907-5912

\section{Introduction}

Upper tract urothelial carcinoma (UTUC) is rare and occupied $5 \%$ of the urothelial carcinoma (Munoz and Ellison, 2000; Siegel et al., 2013). The standard treatment of UTUC is nephroureterectomy (NU) with bladder cuff resection which means one kidney would be lost after this extirpative management. It yields high risk of followed chronic kidney disease and makes following pharmacotherapy treatment restricted because of impaired renal function (Malcolm et al., 2009). Renal function preservation is becoming recognized and kidney-sparing procedures are reserved for selected patients with lowgrade UTUC in EAU guidelines (Roupret et al., 2013). Kidney-sparing management (KS) has been reported with durable prognostic outcomes as nephroureterectomy in several studies (Gadzinski et al., 2010; Jeldres et al., 2010; Cutress et al., 2013; Fukushima et al., 2014). Kidney preservation treatments mainly comprise segmental ureterectomy and endoscopic resection or ablation. With the advance of endoscopic techniques, these conservative managements become popular for UTUC treatment.
However, due to low incidence of UTUC, the paucity of comparative data makes the weak evidence of KS. Herein we summarize the published literatures and perform a meta-analysis to update the evidence of oncologic outcomes of kidney-sparing management.

\section{Materials and Methods}

\section{Search strategy and literature screening}

A literature search of PubMed, Embase, and The Cochrane Library was conducted by two independent investigators (DL She and Y Luo) according to the PICOS principle to retrieve the clinical studies through to December 2014. The search terms used were "ureter cancer", "renal pelvis cancer", "upper tract urothelial carcinoma", "nephroureterectomy", "ureterectomy", "nephron sparing", "survival", "hazard ratio" and "mortality", etc. References in the retrieved literature and previous systematic reviews were also identified for any relevant studies. The eligibility criteria were that any comparative studies simultaneously included both nephroureterectomy and nephron-sparing management

Institute of Gansu Nephro-Urological Clinical Center, Institute of Urology, Department of Urology, Key Laboratory of Urological Disease of Gansu Province, Lanzhou University Second Hospital, Lanzhou, China ${ }^{\star}$ Equal contributors *For correspondence: yuze250@163.com. 


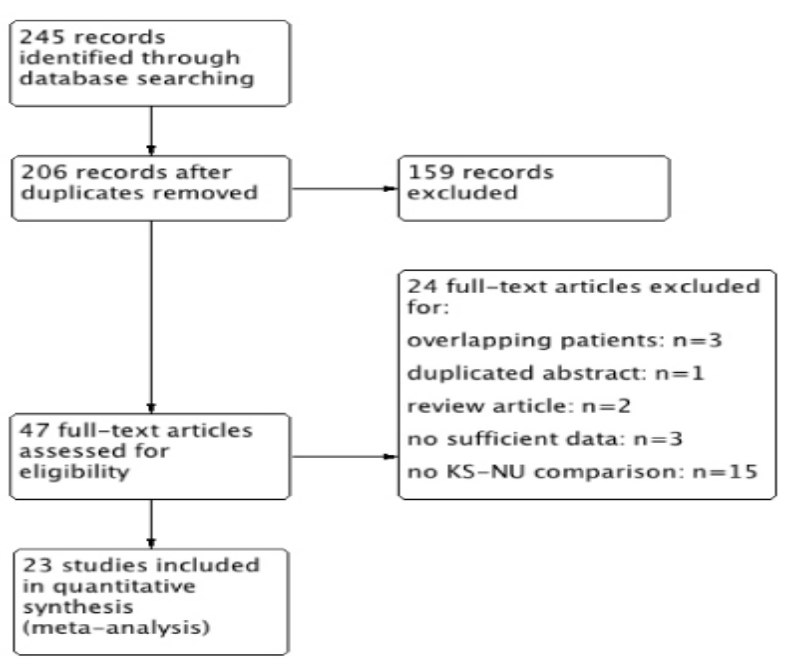

Figure 1. Screening Flow Diagram

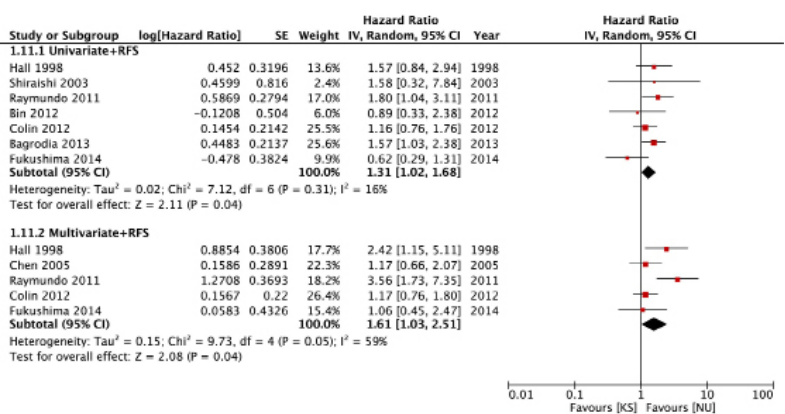

Figure 2. Forest Plot of Intravesical Recurrence Free Survival (IRFS) Hazard Ratio

for upper tract urothelial carcinoma. Any study containing any following criteria was excluded: (1) any study labeled conservative management but actually containing nephrectomy compared with nephroureterectomy; (2) no related data could be extracted or calculated; or (3) duplicate published papers or studies containing overlapping patients. This systematic review was performed in compliance with the Preferred Reporting Items for Systematic Reviews and Meta-Analyses statement (PRISMA) (Moher et al., 2009).

\section{Quality assessment and data extraction}

The quality of the included studies was independently assessed by 2 investigators (Y Luo and DL She) using the Oxford Centre for Evidence-based Medicine Levels of Evidence (March 2009) (OCEBM 2009) (Phillips et al., 2009) and the data extraction was also performed and cross-checked. If there was a discrepancy of opinions or results, it was solved by group discussion. We extracted the name of the first author, year of publication, regions or data sources, number of analyzed patients, age, approaches of kidney-sparing management, cancer category, reported results, follow-up years from the citations. The data were extracted from the reported original data, if possible. When no direct survival data were included, it can be calculated or estimated by using the methods that Tierney et al provided (Tierney et al., 2007), or obtained from related systematic review including this study. Contacting corresponding author was another action for the original data. Studies were excluded if above methods were failure.

\section{Statistical analysis}

Review Manager 5.3 (The Nordic Cochrane Centre, Copenhagen, Denmark) was used to conduct the metaanalysis and Stata 13 (Stata Corp LP, College Station, TX, USA) was used to perform the publication bias detection. Oncologic outcomes included recurrence free survival (RFS), intravesical recurrence free survival (IRFS), metastasis free survival (MFS), cancer specific survival (CSS) and overall survival (OS) using the effect size hazard ratio (HR). First, Cochrane's $Q$ test and $I^{2}$ statistic were calculated for heterogeneity. $P \geq 0.1$ and $\mathrm{I}^{2}<50 \%$ indicated no significant heterogeneity, in which case a fixed model was used. In contrast, a random model was used. Publication bias was detected by the Egger's test for funnel plot asymmetry and $P>0.1$ was considered no significant evidence of publication bias. A 2-tailed $P=0.05$ was set as the significance level.

\section{Results}

\section{Literature filtration and quality assessment}

Two hundred forty-five references were retrieved by the initial search strategy. Screening flow diagram was shown in Figure 1. Of the 47 full-text articles, four references (Lughezzani et al., 2009; Jeldres et al., 2010; Lughezzani et al., 2010; Simhan et al., 2014) were derived from SEER database and had overlapping areas. Hence, we selected the one with more areas and patients. After rounds of screening, twenty-three studies including 1587 KS and $3996 \mathrm{NU}$ patients were enrolled meta-analysis at last (Hall et al., 1998; Shiraishi et al., 2003; Chen et al., 2005; Roupret et al., 2006; Giannarini et al., 2007; Lehmann et al., 2007; Lucas et al., 2008; Dragicevic et al., 2009; Gadzinski et al., 2010; Jeldres et al., 2010; Raymundo et al., 2011; Bin et al., 2012; Bing-bing et al., 2012; Colin et al., 2012; Grasso et al., 2012; Bagrodia et al., 2013; Cutress et al., 2013; Fajkovic et al., 2013; Klatte et al., 2013; Dalpiaz et al., 2014; Fukushima et al., 2014; Hoffman et al., 2014; Hung et al., 2014). All included studies were retrospective comparative study; no randomized controlled trials were identified. Kidney sparing managements included ureteroscopic treatment or endoscopy management, segmental or partial ureterectomy, ablation and open resection. Outcomes included the original reported results and the calculated or estimated data. Quality assessment of included studies was conducted using OCEBM 2009 and all of included studies were "Outcome" research with the level of evidence $2 c$ or 4 . The main characteristics of included studies were shown in Table 1.

\section{Meta-analysis}

Five survival outcomes were analyzed in the metaanalysis. Univariate analysis was considered as a glimpse reference in the survival analysis and multivariate analysis was the authentic result for evaluation of kidney sparing management. In the recurrence free survival analysis (Figure 2), the pooled univariate RFS hazard ratio was $1.31(95 \% \mathrm{CI}, 1.02-1.68, P=0.04)$ by using the random model. Multivariate analysis yielded a pooled RFS HR of $1.61(1.03-2.51, P=0.04)$. Both univariate and multivariate 


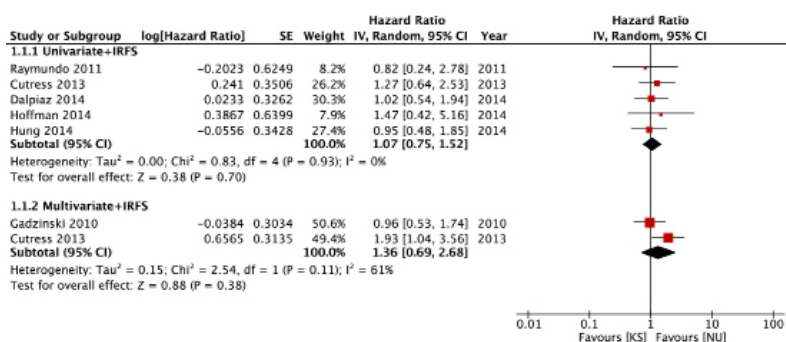

Figure 3. Forest Plot of Recurrence Free Survival (RFS) Hazard Ratio

pooled results showed kidney-sparing management yielded significantly higher risk of tumor recurrence than nephroureterectomy. However, no significant differences were observed in the intravesical recurrence, recurrence hazard ratios of kidney sparing management versus nephroureterectomy were $1.07(0.75-1.52, P=0.70)$ and

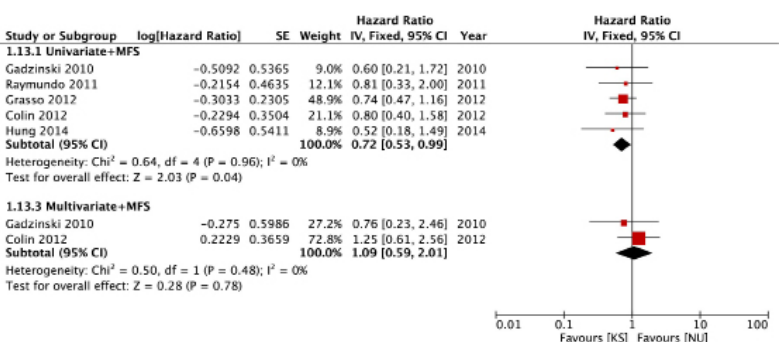

Figure 4. Forest Plot of Metastasis Free Survival (MFS) Hazard Ratio

$1.36(0.69-2.68, P=0.38)$ in univariate and multivariate groups, respectively (Figure 3 ). In the metastasis free survival analysis, pooled univariate HR was 0.72 (0.53$0.99, P=0.04)$, which meant more probability of tumor metastasis was observed in the nephroureterectomy patients. While multivariate pooled outcome of two studies

Table 1. Characteristics of Included Studies Compared KS and NU

\begin{tabular}{|c|c|c|c|c|c|c|c|c|}
\hline Studies & Regions & Duration & $\begin{array}{l}\mathrm{n}(\mathrm{KS} / \\
\mathrm{NU})\end{array}$ & $\begin{array}{c}\text { Age (range) } \\
\text { years }\end{array}$ & $\begin{array}{c}\mathrm{KS} \\
\text { approaches }\end{array}$ & Cancer & $\begin{array}{l}\text { Out- } \\
\text { comes }\end{array}$ & $\begin{array}{l}\text { Follow-up } \\
\text { (range) } \\
\text { months }\end{array}$ \\
\hline Hall 1998 & USA & $\begin{array}{l}1960.1- \\
1992.12\end{array}$ & $42 / 194$ & $(30-95)$ & $\begin{array}{l}\text { DU, SU, } \\
\text { open- } \\
\text { excision, } \\
\text { ablation }\end{array}$ & $\begin{array}{l}\text { UTUC (G1, } \\
\text { G2, G3) }\end{array}$ & RFS CSS & Md64 (1-255) \\
\hline $\begin{array}{l}\text { Shiraishi } \\
2003\end{array}$ & Japan & $\begin{array}{l}1995- \\
2001\end{array}$ & $9 / 10$ & Mn69.7 & PU, ablation & $\begin{array}{l}\text { UTUC (G1, } \\
\text { G2, G3) }\end{array}$ & RFS & NA \\
\hline Chen 2005 & Taiwan & $\begin{array}{l}1993.1- \\
2003.12\end{array}$ & $16 / 78$ & $\begin{array}{c}\text { Mn70.5 (38- } \\
91)\end{array}$ & $\begin{array}{l}\text { SU, ureteros- } \\
\text { copy manage- } \\
\text { ment }\end{array}$ & $\begin{array}{l}\text { Ureter cancer } \\
(\mathrm{G} 1, \mathrm{G} 2, \mathrm{G} 3)\end{array}$ & RFS CSS & $\begin{array}{c}\text { Mn49.3 (1- } \\
136)\end{array}$ \\
\hline $\begin{array}{l}\text { Roupret } \\
2006\end{array}$ & France & $\begin{array}{l}1990- \\
2004\end{array}$ & $43 / 54$ & Mn68 & $\begin{array}{l}\text { PNR, ure- } \\
\text { teroscopic } \\
\text { resection }\end{array}$ & $\begin{array}{l}\text { UTUC (low, } \\
\text { high grade) }\end{array}$ & CSS & Mn54 \\
\hline $\begin{array}{c}\text { Lehmann } \\
2007\end{array}$ & Germany & $\begin{array}{l}1975- \\
2004\end{array}$ & $51 / 91$ & $\begin{array}{c}\text { Md68 (29- } \\
85)\end{array}$ & PU & $\begin{array}{l}\text { Ureter cancer } \\
(\mathrm{G} 1, \mathrm{G} 2, \mathrm{G} 3)\end{array}$ & CSS & Md96 \\
\hline $\begin{array}{c}\text { Giannarini } \\
2007\end{array}$ & Switzerland & $\begin{array}{l}1974.12- \\
2004.12\end{array}$ & $19 / 24$ & $\begin{array}{l}\text { Md72 (31- } \\
86)\end{array}$ & PU & $\begin{array}{c}\text { Distal ureter } \\
\text { cancer (G1, } \\
\text { G2, G3) }\end{array}$ & CSS OS & $\operatorname{Md58}(3-260)$ \\
\hline $\begin{array}{c}\text { Lucas } \\
2008 \\
\end{array}$ & USA & $\begin{array}{l}1990- \\
2005\end{array}$ & $39 / 77$ & $(33-89)$ & PNR & $\begin{array}{l}\text { UTUC (low, } \\
\text { high grade) }\end{array}$ & CSS OS & $\begin{array}{c}\mathrm{Md} 45.8(0.5- \\
129)\end{array}$ \\
\hline $\begin{array}{c}\text { Dragicevic } \\
2009\end{array}$ & Serbia & $\begin{array}{l}1998.1- \\
2002.12\end{array}$ & $21 / 86$ & $\begin{array}{c}\text { Md67 (38- } \\
86)\end{array}$ & PU, ablation & $\begin{array}{l}\text { UTUC (G1, } \\
\text { G2, G3) }\end{array}$ & OS & Md67 (46-88) \\
\hline $\begin{array}{c}\text { Gadzinski } \\
2010\end{array}$ & USA & $\begin{array}{l}1996- \\
2004\end{array}$ & $34 / 62$ & $\begin{array}{c}\text { Mn70 (33.5- } \\
93.2)\end{array}$ & $\begin{array}{l}\text { Endoscopic } \\
\text { management }\end{array}$ & $\begin{array}{l}\text { UTUC (low, } \\
\text { high grade) }\end{array}$ & $\begin{array}{c}\text { IRFS } \\
\text { MFS } \\
\text { CSS OS }\end{array}$ & $\begin{array}{c}\operatorname{Md} 56.6(2.3- \\
146.7)\end{array}$ \\
\hline $\begin{array}{l}\text { Jeldres } \\
2010\end{array}$ & $\begin{array}{c}\text { USA } \\
\text { (SEER) }\end{array}$ & $\begin{array}{l}1988- \\
2006\end{array}$ & $569 / 1222$ & $\begin{array}{l}\text { Mn72 (30- } \\
95)\end{array}$ & SU & $\begin{array}{c}\text { Ureter cancer } \\
(\mathrm{G} 1, \mathrm{G} 2, \mathrm{G} 3, \\
\mathrm{G} 4)\end{array}$ & CSS & Md30 \\
\hline $\begin{array}{c}\text { Raymundo } \\
2011\end{array}$ & USA & $\begin{array}{c}199.1- \\
2009.10\end{array}$ & $21 / 99$ & $\begin{array}{c}\operatorname{Mn} 72.2(37- \\
98)\end{array}$ & $\begin{array}{l}\text { Percutaneous/ } \\
\text { ureteroscopic } \\
\text { resection/ } \\
\text { fulguration, } \\
\text { basketing of } \\
\text { UT-UC }\end{array}$ & $\begin{array}{l}\text { UTUC (G1, } \\
\text { G2, G3) }\end{array}$ & $\begin{array}{l}\text { RFS } \\
\text { IRFS } \\
\text { MFS }\end{array}$ & $\begin{array}{c}\text { Mn17.9 } \\
(13.2-24.6)\end{array}$ \\
\hline Shi 2012 & China & $\begin{array}{l}1997.4- \\
2008.4\end{array}$ & $75 / 64$ & $\begin{array}{c}\text { Mn66.6 (30- } \\
85)\end{array}$ & $\begin{array}{c}\text { SU, } \\
\text { endourologic } \\
\text { management }\end{array}$ & $\begin{array}{l}\text { UTUC (well, } \\
\text { moderate, poor } \\
\text { differentiation) }\end{array}$ & OS & $\begin{array}{c}\text { Md43.4 (0.2- } \\
120)\end{array}$ \\
\hline $\begin{array}{c}\text { Grasso } \\
2012\end{array}$ & USA & $\begin{array}{l}1996.1- \\
2011.8\end{array}$ & $80 / 80$ & $\begin{array}{c}\text { Md73 (45- } \\
93)\end{array}$ & $\begin{array}{l}\text { Ureteroscopy } \\
\text { management }\end{array}$ & $\begin{array}{l}\text { UTUC (low, } \\
\text { high grade) }\end{array}$ & $\begin{array}{c}\text { MFS } \\
\text { CSS OS }\end{array}$ & $\begin{array}{c}\text { Mn38.2 (1- } \\
185.3)\end{array}$ \\
\hline
\end{tabular}




\begin{tabular}{|c|c|c|c|c|c|c|c|c|}
\hline Studies & Regions & Duration & $\begin{array}{l}\mathrm{n}(\mathrm{KS} / \\
\mathrm{NU})\end{array}$ & $\begin{array}{c}\text { Age (range) } \\
\text { years }\end{array}$ & $\begin{array}{c}\text { KS } \\
\text { approaches }\end{array}$ & Cancer & $\begin{array}{l}\text { Out- } \\
\text { comes }\end{array}$ & $\begin{array}{l}\text { Follow-up } \\
\text { (range) } \\
\text { months }\end{array}$ \\
\hline Colin 2012 & France & $\begin{array}{l}1995.1- \\
2009.12\end{array}$ & $52 / 416$ & Md70.1/69.1 & SU & $\begin{array}{l}\text { UTUC (G1, } \\
\text { G2, G3) }\end{array}$ & $\begin{array}{l}\text { RFS } \\
\text { MFS } \\
\text { CSS }\end{array}$ & $\begin{array}{c}\mathrm{Md} 26 \\
\text { (IQR10-48) }\end{array}$ \\
\hline Bin 2012 & USA & $\begin{array}{l}2000.10- \\
2010.12\end{array}$ & $27 / 33$ & $\begin{array}{c}\text { Md73 } \\
\text { (IQR64- } \\
82.8)\end{array}$ & $\begin{array}{c}\mathrm{PU}, \\
\text { endoscopic } \\
\text { resection }\end{array}$ & $\begin{array}{l}\text { Ureter cancer } \\
\text { (low, high } \\
\text { grade) }\end{array}$ & RFS CSS & $\begin{array}{c}\text { Md29 } \\
\text { (IQR11.8- } \\
44.4)\end{array}$ \\
\hline $\begin{array}{c}\text { Bagrodia } \\
2013\end{array}$ & USA & $\begin{array}{l}1992- \\
2006\end{array}$ & $81 / 754$ & $\begin{array}{c}\text { Md69 (32- } \\
97)\end{array}$ & PU & $\begin{array}{l}\text { UTUC (low, } \\
\text { high grade) }\end{array}$ & RFS CSS & $\operatorname{Md} 34(1-246)$ \\
\hline $\begin{array}{c}\text { Cutress } \\
2013\end{array}$ & UK & $\begin{array}{l}1991.1- \\
2011.9\end{array}$ & $59 / 70$ & $\operatorname{Md74.8}$ & $\begin{array}{l}\text { Ureteroscopic } \\
\text { ablation, } \\
\text { percutaneous } \\
\text { resection }\end{array}$ & $\begin{array}{l}\text { UTUC (G1, } \\
\text { G2, G3) }\end{array}$ & $\begin{array}{c}\text { IRFS } \\
\text { CSS OS }\end{array}$ & Md50 \\
\hline $\begin{array}{c}\text { Fajkovic } \\
2013\end{array}$ & Austria & $\begin{array}{l}1996- \\
2012\end{array}$ & $20 / 178$ & Mn69.2 & $\begin{array}{l}\text { Endoscopic } \\
\text { management }\end{array}$ & $\begin{array}{l}\text { UTUC (low, } \\
\text { high grade) }\end{array}$ & CSS OS & Mn20.4 \\
\hline $\begin{array}{l}\text { Klatte } \\
2013\end{array}$ & $\begin{array}{l}\text { European } \\
\text { multicenter }\end{array}$ & NA & $177 / 177$ & NA & SU & Ureter cancer & CSS & Md35 \\
\hline Hung 2014 & Taiwan & $\begin{array}{l}2004.7- \\
2010.8\end{array}$ & $35 / 77$ & Md68 & SU & $\begin{array}{c}\text { Ureter cancer } \\
\text { (low, high } \\
\text { grade) }\end{array}$ & $\begin{array}{l}\text { IRFS } \\
\text { CSS } \\
\text { MFS }\end{array}$ & Mn48.3/43.8 \\
\hline $\begin{array}{c}\text { Hoffman } \\
2014\end{array}$ & Israel & $\begin{array}{l}200- \\
2010\end{array}$ & $25 / 22$ & Md64/76 & $\begin{array}{l}\text { Endoscopic } \\
\text { resection }\end{array}$ & $\begin{array}{l}\text { UTUC (low } \\
\text { grade) }\end{array}$ & IRFS OS & $\operatorname{Md} 26 / 57$ \\
\hline $\begin{array}{c}\text { Fukushima } \\
2014\end{array}$ & Japan & 1977-NA & $43 / 86$ & NA & $\begin{array}{c}\text { Distal } \\
\text { ureterectomy }\end{array}$ & $\begin{array}{c}\text { Distal ureter } \\
\text { cancer }(\mathrm{G} 1 \text {, } \\
\text { G2, G3) }\end{array}$ & RFS CSS & $\begin{array}{l}\text { Md50 (16- } \\
103)\end{array}$ \\
\hline $\begin{array}{c}\text { Dalpiaz } \\
2014\end{array}$ & Austria & $\begin{array}{l}1984.1- \\
2011.3\end{array}$ & $49 / 42$ & $\begin{array}{c}\operatorname{Md} 71.5 / 70.3 \\
(49-88)\end{array}$ & $\begin{array}{c}\text { Distal } \\
\text { ureterectomy }\end{array}$ & $\begin{array}{c}\text { Distal ureter } \\
\text { cancer (G1, } \\
\text { G2, G3) }\end{array}$ & $\begin{array}{c}\text { IRFS } \\
\text { CSS OS }\end{array}$ & $\begin{array}{c}\operatorname{Md} 51.1 / 51.5 \\
\quad(4-290)\end{array}$ \\
\hline
\end{tabular}

*SEER=Surveillance, Epidemiology and End Results database; KS=kidney sparing; $\mathrm{SU}=$ segmental ureterectomy; PU=partial ureterectomy; DU=distal ureterectomy; PNR=percutaneous nephroscopic resection; Mn=mean; Md=median; UTUC=upper tract urothelial carcinoma; IQR=interquartile range; $\mathrm{NA}=$ not applicable

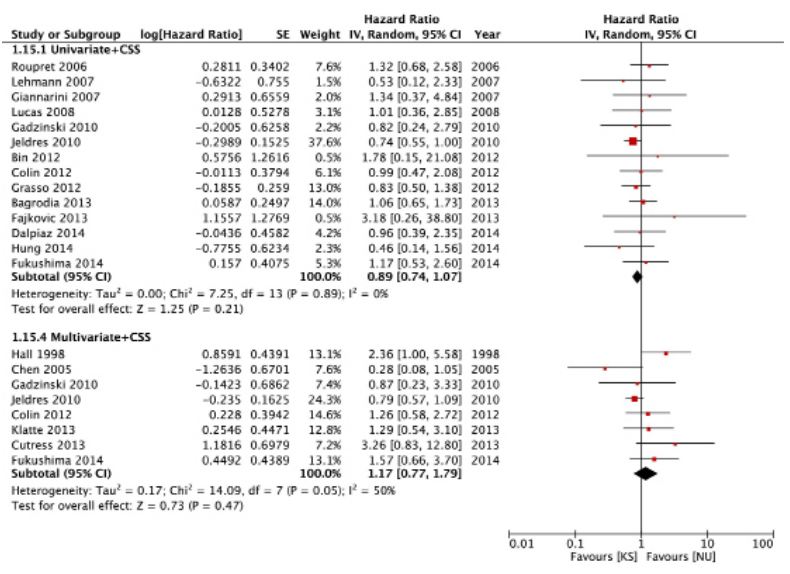

Figure 5. Forest Plot of Cancer Specific Survival (CSS) Hazard Ratio

of $1.09(0.59-2.01, P=0.78)$ did not validate this result, temporarily (Figure 4). Univariate cancer specific survival was acquired from 11 studies and multivariate outcomes were from 8 studies. Both of pooled outcomes revealed equivalent cancer mortality risk based on the hazard ratio of $0.89(0.74-1.07, P=0.21)$ in univariate $\mathrm{CSS}$ and $1.17(0.77-1.79, P=0.47)$ in multivariate CSS (Figure 5). Additionally, no significant overall survival differences were observed from the pooled OS outcomes in univariate

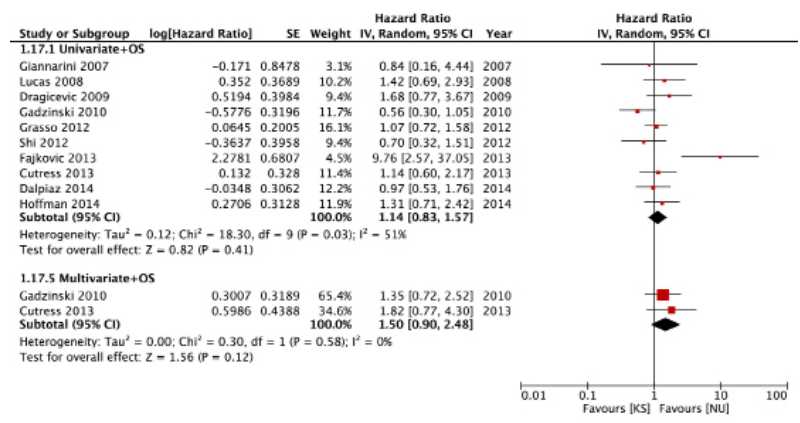

Figure 6. Forest Plot of Overall Survival (OS) Hazard Ratio

hazard of $1.14(0.83-1.57, P=0.41)$ and multivariate hazard of $1.50(0.90-2.48, P=0.12)$ (Figure 6).

Publication bias and sensitive analysis

To assure compliance with Cochrane handbook recommendations, Egger's test for funnel plot asymmetry was conducted in meta-analysis included at least ten studies as a rule of thumb (Higgins and Green, 2011). Hence, we performed this test in CSS meta-analysis. The $P$ value of Egger's test in the univariate CSS, multivariate CSS group were 0.158 and 0.298 , respectively. No significant evidence of publication bias was observed 
(funnel plots were omitted).

We performed leave-one-out sensitive analysis to detect any single study affected systematic result and effect models alteration to check the feasibility of the pooled result. No pooled result was altered using sensitive analysis (results were omitted) except RFS and univariate MFS which were "marginal significant". It showed that the conclusion kidney-sparing management had more tumor recurrence risk than nephroureterectomy was not feasible and could easily be altered by following few studies. Therefore, more studies were needed to validate the recurrence risk between kidney-sparing management and nephroureterectomy.

\section{Discussion}

Our meta-analysis enrolled twenty-three studies that contained various oncologic outcomes and majority of the pooled results favored kidney sparing management as an alteration for upper tract urothelial carcinoma with equivalent survival outcomes as nephroureterectomy. However, this was based on low level of evidences and no stage or grade stratification was conducted. Many factors affected the survival to different extent. Seisen et al (Seisen et al., 2014) summarized clinicopathologic factors on intravesical recurrence after nephroureterectomy in upper tract urothelial carcinoma. Of the results gender, prior bladder cancer, patient with chronic kidney disease were the risk factors, and tumor related factors such as positive cytology, tumor location, multifocality, invasive pT stage, and necrosis were significant recurrence predictors.Additionally, laparoscopic nephroureterectomy, extravesical bladder cuff resection and positive surgical margin were also the predictors. Previous ureteroscopy seemed to be an independent intravesical recurrence risk factor (Luo et al., 2013), but it's still controversial (Ishikawa et al., 2010; Gurbuz et al., 2011; Nison et al., 2013). Tumor grade was another impact factor of oncologic outcome. Cutress et al (Cutress et al., 2013) found that compared with nephroureterectomy, kidney preservation treatment had significantly lower survival rate of IRFS, PFS, CSS and OS in G2 and G3 but equivalent in G1. On the other hand, grade 2 and grade 3 upper tract urothelial carcinoma was significantly poor compared with grade 1 (Grasso et al., 2012).

As a compromise, initial conservative management with regular surveillance and followed extirpative procedure for disease advance could be a sufficient solution. Recent reports suggest that delayed nephroureterectomy do not affect the oncologic survival significantly (Boorjian et al., 2005; Gadzinski et al., 2012; Sundi et al., 2012). Initial ureteroscopic ablation didn't affect the recurrence and cancer specific mortality after radical nephroureterectomy with the hazard ratio of $0.79, P=0.185$ and $0.7, P=0.078$, respectively (Gurbuz et al., 2011). Yakoubi et al summarized previously published comparative reports of endoscopic procedure and nephroureterectomy and preliminarily concluded equivalent overall and cancer specific survival based on the low level of evidences (Yakoubi et al., 2014). Our meta-analysis was compliance with these results. It's safe to apply endoscopic procedures for initial management of upper tract urothelial carcinoma. However, up-stage and progression as a result of delayed extirpative treatment may damage the following nephroureterectomy (Waldert et al., 2010).

Though above results showed no significant survival advantage using nephroureterectomy versus using kidney preservation treatment. It cannot compellingly conclude that kidney preservation procedure provided equivalent tumor control in all upper tract urothelial carcinoma patients. Because this equivalent effect probably was contributed by low grade or low stage upper tract urothelial carcinoma, paucity of the number, low quality of included studies and selection bias (though multivariate analysis was conducted). Our results merely provided a comprehensive outcome and should be interpreted with caution. No more detail information such as grade or stage stratification that could give a direct guidance for clinical practice was provided. Hence, kidney preservation management may be only conservatively applied in low-grade upper tract urothelial carcinoma for now. Additionally, considering the low incidence rate of upper tract urothelial carcinoma, multi-institute randomized controlled trials are needed for further verification.

In conclusion, kidney-sparing management had equivalent prognostic effect on upper tract urothelial carcinoma as the standard nephroureterectomy except in tumor recurrence, overall. But the results should be interpreted with cautions for lack of stage and grade stratification and multi-center randomized controlled trials are still needed to further verify our results.

\section{References}

Bagrodia A, Kuehhas FE, Gayed BA, et al (2013). Comparative analysis of oncologic outcomes of partial ureterectomy vs radical nephroureterectomy in upper tract urothelial carcinoma. Urology, 81, 972-7.

Bin X, Roy OP, Ghiraldi E, et al (2012). Impact of tumour location and surgical approach on recurrence-free and cancer-specific survival analysis in patients with ureteric tumours. BJU Int, 110, 514-9.

Bing-bing S, Cheng C, Han-zhong L, et al (2012). Correlation between surgical modality and clinicopathologic characteristics for ureteral transitional cell carcinoma. Clin Transl Oncol, 14, 312-6.

Boorjian S, Ng C, Munver R, et al (2005). Impact of delay to nephroureterectomy for patients undergoing ureteroscopic biopsy and laser tumor ablation of upper tract transitional cell carcinoma. Urology, 66, 283-7.

Chen WJ, Kuo JY, Chen KK, et al (2005). Primary urothelial carcinoma of the ureter: 11-year experience in Taipei veterans general hospital. J Chin Med Assoc, 68, 522-30.

Colin $P$, Ouzzane A, Pignot G, et al (2012). Comparison of oncological outcomes after segmental ureterectomy or radical nephroureterectomy in urothelial carcinomas of the upper urinary tract: results from a large French multicentre study. BJU Int, 110, 1134-41.

Cutress ML, Stewart GD, Tudor EC, et al (2013). Endoscopic versus laparoscopic management of noninvasive upper tract urothelial carcinoma: 20-year single center experience. J Urol, 189, 2054-60.

Dalpiaz O, Ehrlich G, Quehenberger F, et al (2014). Distal ureterectomy is a safe surgical option in patients with urothelial carcinoma of the distal ureter. Urol Oncol, 32, 1-8. 
Dragicevic D, Djokic M, Pekmezovic T, et al (2009). Comparison of open nephroureterectomy and open conservative management of upper urinary tract transitional cell carcinoma. Urol Int, 82, 335-40.

Fajkovic H, Klatte T, Nagele U, et al (2013). Results and outcomes after endoscopic treatment of upper urinary tract carcinoma: the Austrian experience. World J Urol, 31, 37-44.

Fukushima H, Saito K, Ishioka J, et al (2014). Equivalent survival and improved preservation of renal function after distal ureterectomy compared with nephroureterectomy in patients with urothelial carcinoma of the distal ureter: a propensity score-matched multicenter study. Int J Urol, 21, 1098-104.

Gadzinski AJ, Roberts WW, Faerber GJ, et al (2010). Longterm outcomes of nephroureterectomy versus endoscopic management for upper tract urothelial carcinoma. J Urol, 183, 2148-53.

Gadzinski AJ, Roberts WW, Faerber GJ, et al (2012). Long-term outcomes of immediate versus delayed nephroureterectomy for upper tract urothelial carcinoma. J Endourol, 26, 566-73.

Giannarini G, Schumacher MC, Thalmann GN, et al (2007). Elective management of transitional cell carcinoma of the distal ureter: can kidney-sparing surgery be advised? BJU Int, 100, 264-8.

Grasso M, Fishman AI, Cohen J, et al (2012). Ureteroscopic and extirpative treatment of upper urinary tract urothelial carcinoma: a 15-year comprehensive review of 160 consecutive patients. BJU Int, 110, 1618-26.

Gurbuz C, Youssef RF, Shariat SF, et al (2011). The impact of previous ureteroscopic tumor ablation on oncologic outcomes after radical nephrouretectomy for upper urinary tract urothelial carcinoma. J Endourol, 25, 775-9.

Hall MC, Womack S, Sagalowsky AI, et al (1998). Prognostic factors, recurrence, and survival in transitional cell carcinoma of the upper urinary tract: a 30 -year experience in 252 patients. Urol, 52, 594-601.

Higgins JPT, Green S (2011). Cochrane Handbook for Systematic Reviews of Interventions Version 5.1.0 [updated March 2011] [Online]. The Cochrane Collaboration. Available: www. cochrane-handbook.org.

Hoffman A, Yossepowitch O, Erlich Y, et al (2014). Oncologic results of nephron sparing endoscopic approach for upper tract low grade transitional cell carcinoma in comparison to nephroureterectomy - a case control study. BMC Urol, 14, 97.

Hung SY, Yang WC, Luo HL, et al (2014). Segmental ureterectomy does not compromise the oncologic outcome compared with nephroureterectomy for pure ureter cancer. Int Urol Nephrol, 46, 921-6.

Ishikawa S, Abe T, Shinohara N, et al (2010). Impact of diagnostic ureteroscopy on intravesical recurrence and survival in patients with urothelial carcinoma of the upper urinary tract. J Urol, 184, 883-7.

Jeldres C, Lughezzani G, Sun M, et al (2010). Segmental ureterectomy can safely be performed in patients with transitional cell carcinoma of the ureter. J Urol, 183, 1324-9.

Klatte T, Zigeuner RE, Rouprêt M, et al (2013). 575 Segmental ureterectomy versus radical nephroureterectomy for urothelial cancer of the ureter: A matched-pair analysis. Eur Urol Suppl, 12, 575-6.

Lehmann J, Suttmann H, Kovac I, et al (2007). Transitional cell carcinoma of the ureter: prognostic factors influencing progression and survival. Eur Urol, 51, 1281-8.

Lucas SM, Svatek RS, Olgin G, et al (2008). Conservative management in selected patients with upper tract urothelial carcinoma compares favourably with early radical surgery. BJU Int, 102, 172-6.

Lughezzani G, Jeldres C, Isbarn H, et al (2009). Nephroureterectomy and segmental ureterectomy in the treatment of invasive upper tract urothelial carcinoma: a population-based study of 2299 patients. Eur J Cancer, 45, 3291-7.
Lughezzani G, Jeldres C, Isbarn H, et al (2010). Temporal stage and grade migration in surgically treated patients with upper tract urothelial carcinoma. BJU Int, 105, 799-804.

Luo HL, Kang CH, Chen YT, et al (2013). Diagnostic ureteroscopy independently correlates with intravesical recurrence after nephroureterectomy for upper urinary tract urothelial carcinoma. Ann Surg Oncol, 20, 3121-6.

Malcolm JB, Bagrodia A, Derweesh IH, et al (2009). Comparison of rates and risk factors for developing chronic renal insufficiency, proteinuria and metabolic acidosis after radical or partial nephrectomy. BJU Int, 104, 476-81.

Moher D, Liberati A, Tetzlaff J, et al (2009). Preferred reporting items for systematic reviews and meta-analyses: the PRISMA statement. PLoS Med, 6, 1000097.

Munoz JJ, Ellison LM (2000). Upper tract urothelial neoplasms: incidence and survival during the last 2 decades. J Urol, 164, 1523-5.

Nison L, Roupret M, Bozzini G, et al (2013). The oncologic impact of a delay between diagnosis and radical nephroureterectomy due to diagnostic ureteroscopy in upper urinary tract urothelial carcinomas: results from a large collaborative database. World J Urol, 31, 69-76.

Phillips B, Ball C, Sackett D, et al (2009). Oxford Centre for Evidence-based Medicine Levels of Evidence (March 2009) [Online]. OCEBM Levels of Evidence Working Group. Available: http://www.cebm.net/oxford-centre-evidencebased-medicine-levels-evidence-march-2009/.

Raymundo EM, Lipkin ME, Banez LB, et al (2011). Third prize: the role of endoscopic nephron-sparing surgery in the management of upper tract urothelial carcinoma. $J$ Endourol, 25, 377-84.

Roupret M, Babjuk M, Comperat E, et al (2013). European guidelines on upper tract urothelial carcinomas: 2013 update. Eur Urol, 63, 1059-71.

Roupret M, Hupertan V, Traxer O, et al (2006). Comparison of open nephroureterectomy and ureteroscopic and percutaneous management of upper urinary tract transitional cell carcinoma. Urol, 67, 1181-7.

Seisen T, Granger B, Colin $P$, et al (2014). A Systematic Review and Meta-analysis of Clinicopathologic Factors Linked to Intravesical Recurrence After Radical Nephroureterectomy to Treat Upper Tract Urothelial Carcinoma. Eur Urol.

Shiraishi K, Eguchi S, Mohri J, et al (2003). Role of ureteroscopic biopsy in the management of upper urinary tract malignancy. Int J Urol, 10, 627-30.

Siegel R, Naishadham D, Jemal A (2013). Cancer statistics, 2013. CA Cancer J Clin, 63, 11-30.

Simhan J, Smaldone MC, Egleston BL, et al (2014). Nephronsparing management vs radical nephroureterectomy for low- or moderate-grade, low-stage upper tract urothelial carcinoma. BJU Int, 114, 216-20.

Sundi D, Svatek RS, Margulis V, et al (2012). Upper tract urothelial carcinoma: impact of time to surgery. Urol Oncol, 30, 266-72.

Tierney JF, Stewart LA, Ghersi D, et al (2007). Practical methods for incorporating summary time-to-event data into metaanalysis. Trials, 8, 16-.

Waldert M, Karakiewicz PI, Raman JD, et al (2010). A delay in radical nephroureterectomy can lead to upstaging. BJU Int, 105, 812-7.

Yakoubi R, Colin $P$, Seisen T, et al (2014). Radical nephroureterectomy versus endoscopic procedures for the treatment of localised upper tract urothelial carcinoma: a meta-analysis and a systematic review of current evidence from comparative studies. Eur J Surg Oncol, 40, 1629-34. 